\title{
Alternative Flicker Glass: A New Anti- Suppression Approach to the Treatment of Anisometropic Amblyopia
}

\author{
Ying Yuan ${ }^{a, b}$ Chengcheng Zhu ${ }^{c, d}$ Peng Wang ${ }^{a, e}$ Xiaojun Hu ${ }^{b}$ Wenbo Yao ${ }^{f}$ \\ Xinhui Huang ${ }^{f}$ Bilian Ke $\mathrm{e}^{\mathrm{a}, \mathrm{b}}$ \\ aDepartment of Ophthalmology, Shanghai General Hospital, Shanghai Jiao Tong University School of Medicine, \\ Shanghai, China; 'bational Clinical Research Center for Eye Diseases, Shanghai, China; 'Shanghai Key Laboratory of \\ Fundus Disease, Shanghai, China; 'dShanghai Engineering Center for Visual Science and Photomedicine, Shanghai, \\ China; 'Shanghai Engineering Center for Precise Diagnosis and Treatment of Eye Diseases, Shanghai, China; \\ fShanghai Eye Disease Prevention and Treatment Center, Shanghai, China
}

\section{Keywords}

Alternative flicker glass · Anisometropic amblyopia ·

Contrast sensitivity $\cdot$ Stereoacuity

\begin{abstract}
Introduction: Amblyopia always presents with monocular and binocular dysfunction. In this study, we aim to investigate the efficacy of alternative occlusion using liquid crystal glasses versus continuous occlusion therapy using traditional patches for treating amblyopia. Methods: Eligible subjects with anisometropic amblyopia were randomized into 2 groups: alternative flicker glass (AFG) or patching group. In the AFG group, subjects were instructed to wear the flicker glasses for $1 \mathrm{~h}$ a day. The AFG is a lightweight spectacle frame with liquid crystal lenses that provide direct square-wave alternating occlusion, which were preprogrammed at a temporal frequency of $7 \mathrm{~Hz}$. In the patching group, the patients were prescribed to wear traditional patches for $2 \mathrm{~h}$ a day. The best-corrected visual acuity (BCVA), contrast sensitivity function (CSF), and stereoacuity were measured at the baseline
\end{abstract}

karger@karger.com www.karger.com/ore

Karger $\stackrel{\text { ' }}{5}$
(C) 2021 The Author(s)

Published by S. Karger AG, Basel

This is an Open Access article licensed under the Creative Commons Attribution-NonCommercial-4.0 International License (CC BY-NC) (http://www.karger.com/Services/OpenAccessLicense), applicable to the online version of the article only. Usage and distribution for commercial purposes requires written permission. and 3 and 12 weeks. Results: In this pilot study, a total of 40 children were recruited, with 20 in the AFG group. Mean BCVA improved by $0.17 \pm 0.14 \log M A R(95 \% \mathrm{Cl}=0.10-0.23)$ in the AFG group and $0.18 \pm 0.18 \log M A R(95 \% \mathrm{Cl}=0.09$ $0.26)$ in the patching group from baseline to 12 weeks. The improvement in BCVA in both groups was significant (both $p<0.01$ ), while there was no significant difference between the groups $(p=0.82)$. The CSF of both low and high spatial frequencies exhibited significant improvement at 12 weeks in the AFG group ( $p<0.01$, respectively) and just had a significant improvement at low spatial frequency in the patching group $(p<0.01)$. The stereoacuity significantly improved by $504.00 \pm 848.00(95 \% \mathrm{Cl}=107.12$ to 900.88$)$ arc seconds in the AFG group $(p<0.05)$, while it was $263.50 \pm 639.55$ (95\% $\mathrm{Cl}=-35.82$ to 562.82 ) arc seconds in the patching group ( $p>$ 0.05). Conclusion: Alternative flicker glass was effective in improving both monocular and binocular function, which was most likely achieved by reducing suppression and promoting binocular fusion. This therapy exhibited promise as an alternative method for amblyopia treatment.

(C) 2021 The Author(s).

Published by S. Karger AG, Basel
Correspondence to:

Bilian Ke, kebilian@126.com 


\section{Introduction}

Amblyopia is a neurodevelopmental disorder of the visual cortex, which presents with monocular and binocular dysfunction. This disorder is usually associated with abnormal visual experience, such as anisometropia or strabismus, during early childhood. Traditional amblyopia treatment, including patching or penalization of the sound eye, aimed at the recovery of monocular function [1]. However, other visual functions remain deficient in clinically treated amblyopia such as contrast sensitivity at high spatial frequencies and stereoacuity $[2,3]$. It also had a high risk of recurrence when the treatment was stopped, which may be associated with the residual abnormal binocular vision after monocular treatment $[4,5]$. Additionally, traditional patching treatment for amblyopia had a low compliance for the psychosocial side effects [6]. Therefore, a new treatment method that could not only improve visual acuity of the amblyopic eye but also provide repeated binocular visual experience is needed.

Our understanding of the visual deficits in amblyopia has changed in recent years [7]. It was believed that the visual input from the amblyopic eye was suppressed, resulting in monocular and binocular vision deficits in amblyopia. In support of this, several studies have found that both the monocular and binocular function of the amblyopia could improve when the input in the sound eye was artificially attenuated [8-10]. The adult amblyopic cortex had the potential to improve after perceptual learning, although it was outside of the critical period of visual development [11]. Additionally, the visual acuity in amblyopia of the animals could be restored when the inhibition of the visual cortex was reduced [12]. Together, all of these studies on humans and animals suggested that the vision deficit in amblyopia was caused by the suppression mechanisms in the visual cortex rather than a loss of cellular function. Furthermore, the relationship between the degree of amblyopia and strength of suppression was also investigated [2]. It was found that the stronger the suppression, the greater the difference between the interocular acuity and poorer binocular function $[7,13]$. According to this view, anti-suppression and promoting binocular function should be the key point for treating amblyopia.

In this study, we used a novel treatment that targeted binocular interaction in amblyopia, which presumably decreases the suppression of amblyopia. The alternative flicker glass (AFG) is an electronic LCD display, which could alternatively present images to the sound and amblyopic eyes at a customized rate. The flicker frequency was preprogrammed at $7 \mathrm{~Hz}$, which has been shown to be beyond the time course for interocular suppression in amblyopia [14]. It allowed the subjects to experience binocular vision in such an alternative flicking rate. Therefore, the goal of this study was to evaluate whether the AFG, with 7-Hz flicker therapy, could promote monocular and binocular function. In this report, we evaluated the best-corrected visual acuity (BCVA), contrast sensitivity function (CSF), and stereoacuity before and after the treatment.

\section{Materials and Methods}

Subjects

Eligible children with anisometropic amblyopia, from 7 to 13 years old, who met with the inclusion criteria were enrolled in the study. The children were outpatients at the Ophthalmology Unit of Shanghai General Hospital. The study protocol complied with the requirements of the Institutional Review Board of Shanghai General Hospital, Shanghai Jiao Tong University School of Medicine, and adhered to the tenets of the Declaration of Helsinki. Informed written consent was obtained from the subjects and at least one of the subject's legal guardians before participation. Forty subjects underwent a complete ophthalmic examination including cycloplegic retinoscopy, fundoscopy, and slit-lamp biomicroscopy before entering the study. The inclusion criteria were a BCVA of $0.70 \log$ MAR to $0.20 \log$ MAR in the amblyopic eye, with 2 lines or more of difference between the 2 eyes, and no amblyopia treatment 3 months prior to the study except refractive correction. The subjects had been wearing spectacle correction for 12 weeks prior to the study. Those with intraocular surgery, high myopia (>-6.00D) or hyperopia $>+9.00 \mathrm{D}$ ), ocular disease as the cause of the reduced visual acuity, strabismus, or a family or personal history of seizures were excluded. Anisometropia was defined as an interocular spherical refractive error difference of $1.50 \mathrm{D}$ or more or a cylindrical difference of 1.00D or more.

Device

The AFG is a spectacle frame with lenses of liquid crystal glass, provided by EyeTronix, Inc. The liquid crystal lenses comprise a gel with organic molecules suspended in between 2 thin plates coated with thin polarized film. An electronic shutter controlled by a preprogrammed microchip allows for accurate and fast alternating rate of occlusion. In this study, the flicker rate is set at $7 \mathrm{~Hz}$ with a $50 \%$ duty cycle between the right and left eyes. In the patching group, the nonamblyopic eye was prescribed to wear occlusion patches combined with optimal refractive correction (if needed).

Treatment Procedure and Follow-Up Visits

Each subject was randomly assigned to the AFG or patching group. In the AFG group, subjects were asked to wear the AFG for $1 \mathrm{~h}$ a day, 7 days a week. Additional prescribed corrective glasses were worn when using AFG treatment. In the patching group, subjects were asked to wear the patches for $2 \mathrm{~h}$ a day, 7 days a week. Follow-up visits were conducted at 3 and 12 weeks after treatment. Each subject underwent a comprehensive ophthalmological exam- 
Table 1. The basic characteristics of the subjects

\begin{tabular}{lcc}
\hline & $\begin{array}{l}\text { AFG group } \\
(N=20)\end{array}$ & $\begin{array}{l}\text { Patching group } \\
(N=20)\end{array}$ \\
\hline Gender (F/M) & $10 / 10$ & $8 / 12$ \\
Age, years & $10.00 \pm 2.13$ & $9.05 \pm 2.09$ \\
SE, D & $3.43 \pm 2.21$ & $4.17 \pm 2.47$ \\
BCVA, logMAR & $0.45 \pm 0.20$ & $0.53 \pm 0.25$ \\
Contrast sensitivity & & \\
$\quad$ 3, dyl/deg & $19.8 \pm 13.67$ & $16.45 \pm 10.54$ \\
6, dyl/deg & $20.00 \pm 16.40$ & $25.20 \pm 17.38$ \\
12, dyl/deg & $5.85 \pm 7.72$ & $7.20 \pm 7.00$ \\
18, dyl/deg & $2.20 \pm 1.97$ & $1.98 \pm 0.77$ \\
Stereoacuity, arc seconds & $609.00 \pm 860.54$ & $576.00 \pm 637.15$ \\
\hline \multicolumn{2}{c}{ SE, spherical equivalent; BCVA, best-corrected visual acuity. }
\end{tabular}

ination, including BCVA (logMAR chart), CSF (CSV-1000E charts; Vector Vision), and stereoacuity (Titmus test) at baseline as well as in the following scheduled visits. The Titmus test consists of 9 circles ranging from 800 arc seconds to 40 arc seconds. The test starts with the gross stereoacuity, and inability to correctly identify the fly was recorded as "nil" stereoacuity.

\section{Statistical Analysis}

Subjects who completed each follow-up visit were included in the analysis. The variables were presented as mean \pm standard deviation. Participants with nil stereoacuity ( $>3,000$ arc seconds) were recorded as 3,000 for the purpose of analysis. All statistical evaluations were performed using SAS version 9.2 (SAS Institute). A paired $t$ test was used to assess BCVA, CSF, and stereoacuity before and after treatment for each group. An independent $t$ test was used to analyze BCVA, CSF, and stereoacuity improvement between the AFG and patching groups. $p<0.05$ was considered statistically significant. All tests were 2-tailed.

\section{Results}

\section{Visual Acuity}

Of the 40 children recruited, 20 subjects were randomly assigned to the AFG group and 20 subjects to the patching group. Baseline clinical parameters were similar in both groups (shown in Table 1). After AFG therapy, there was a significant improvement in BCVA (shown in Fig. 1). At the baseline, the mean BCVA was $0.45 \pm 0.20$ $\log$ MAR $(95 \% \mathrm{CI}=0.35-0.54)$, while at 3 and 12 weeks, it improved to $0.37 \pm 0.20 \log \mathrm{MAR}(95 \% \mathrm{CI}=0.27-0.46)$ and $0.28 \pm 0.19 \log$ MAR $(95 \% \mathrm{CI}=0.19-0.37)$ (both $p<$ 0.01 , compared to the baseline) in the AFG group. In the patching group, the mean BCVA improved to $0.43 \pm 0.20$ $\log$ MAR $(95 \% \mathrm{CI}=0.34-0.52)$ and $0.35 \pm 0.18 \log \mathrm{MAR}$

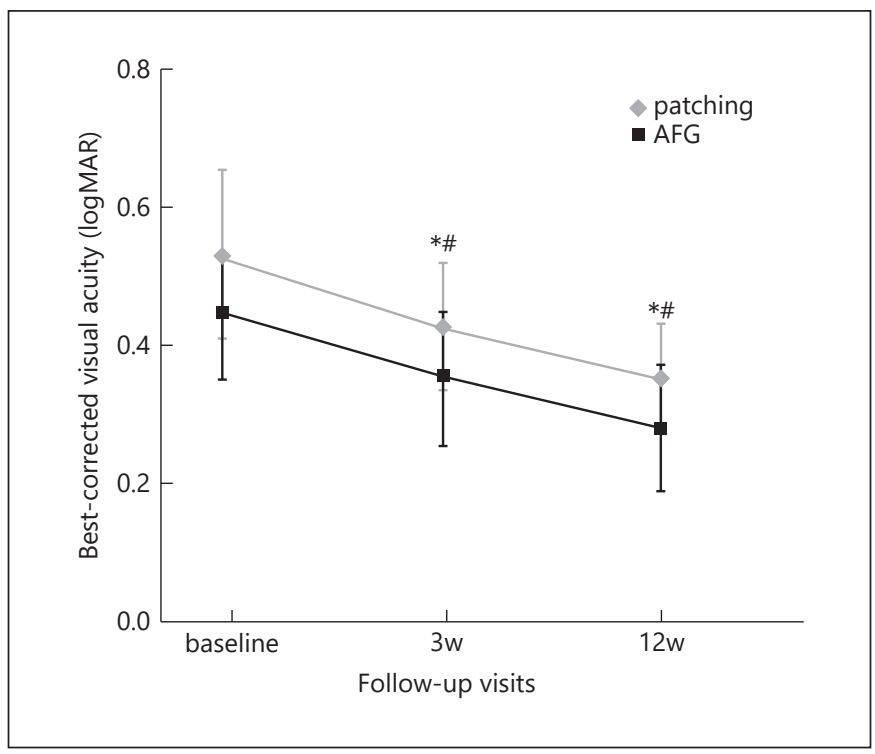

Fig. 1. The best-corrected visual acuity at baseline and each followup visit. Boxes indicate the mean value; whiskers represent the $95 \%$ confidence interval; ${ }^{*} p<0.05$ compared with the baseline visual acuity in the AFG group; ${ }^{*} p<0.05$ compared with the baseline in the patching group.

$(95 \% \mathrm{CI}=0.27-0.43)$ at 3 and 12 weeks, which was significantly different from the baseline $(0.53 \pm 0.25$ logMAR [95\% CI $=0.41-0.65], p<0.01, p<0.05$, shown in Fig. 1). There was no significant difference in BCVA between AFG group and the patching group at baseline and the scheduled follow-up visits $(p=0.25,0.30$, and 0.24 , respectively).

\section{Contrast Sensitivity Function}

Furthermore, we tested the CSF of 3, 6, 12, and $18 \mathrm{cy}$ cles per degree spatial frequencies at baseline and the follow-up visits (shown in Fig. 2). When compared with the baseline, the CSF of all spatial frequencies showed a significant improvement, except for 18 cycles per degree spatial frequency at the visit of 3 weeks in the AFG group $(p<0.01, p<0.01, p<0.01$, and $p=0.07)$. At the 12 -week visit, the CSF of 18 cycles per degree spatial frequency had a significant improvement ( $p<0.01$, compared with the baseline). In the patching group, there was a significant improvement in CSF only in the spatial frequency of 3 cycles per degree at the visits of 3 and 12 weeks (both $p<$ 0.01 ). The improvement in CSF of 6,12 , and 18 cycles per degree spatial frequencies was larger in the AFG group than that in the patching group $(p<0.01, p<0.01$, and $p<0.05$, shown in Fig. 3 ). 

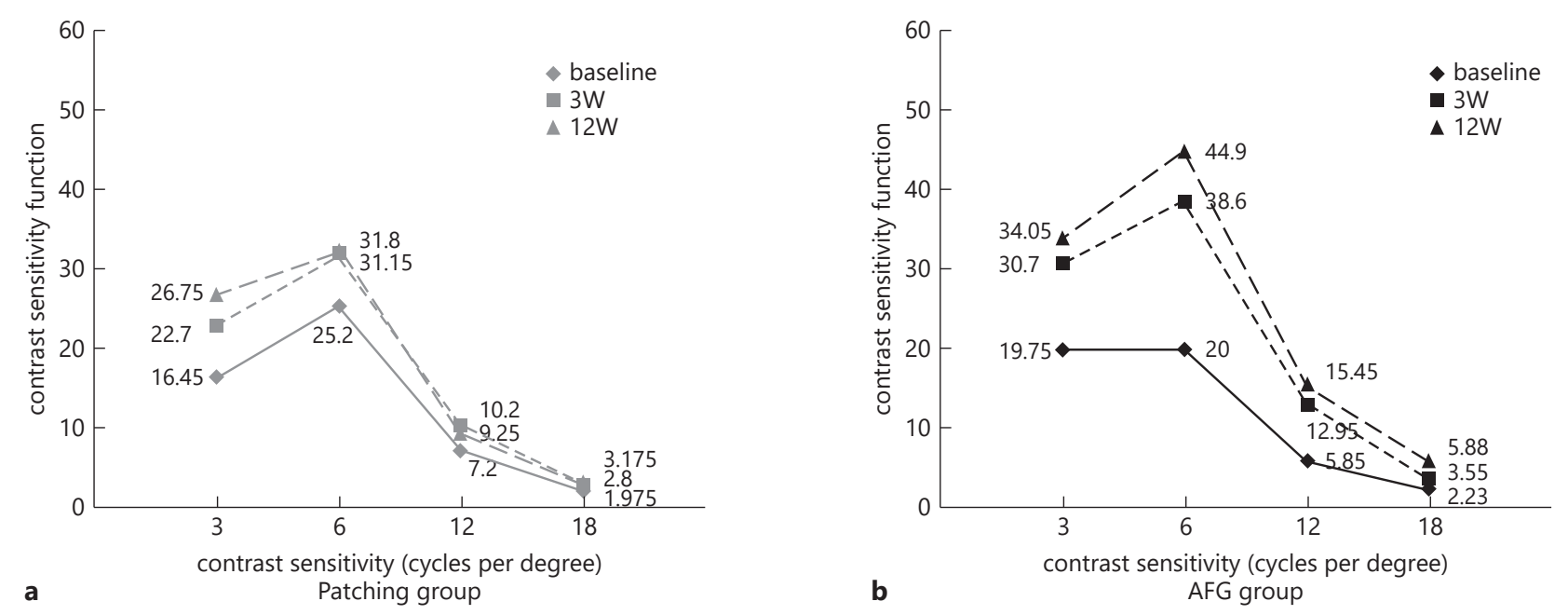

Fig. 2. The contrast sensitivity of the $3,6,12$, and 18 cycles per degree spatial frequencies at baseline and each follow-up visit. a The patching group. $\mathbf{b}$ The AFG group. Boxes indicate the mean value.

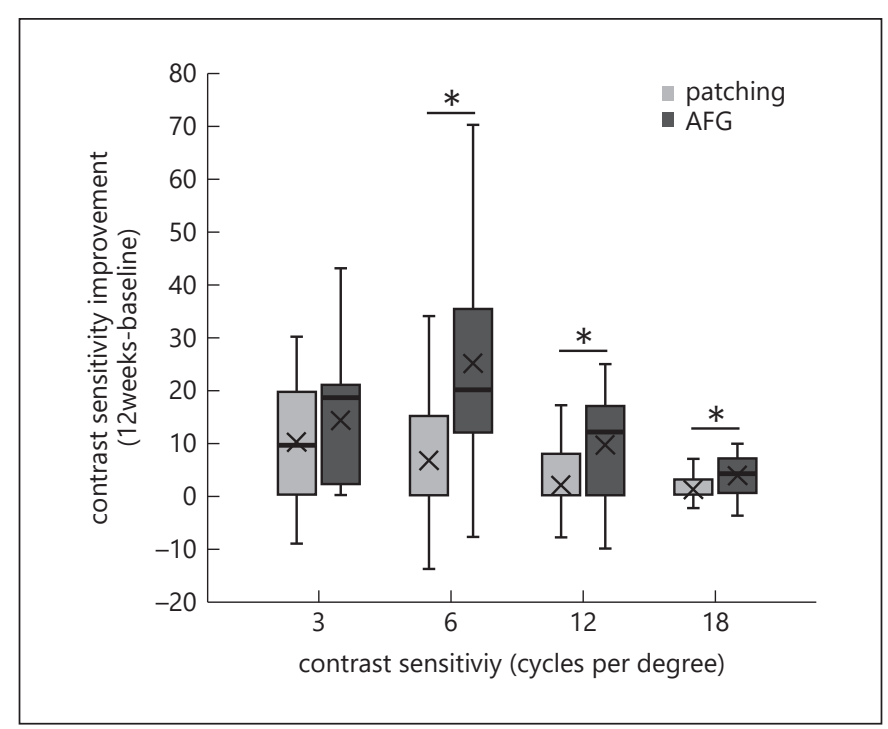

Fig. 3. The improvement in contrast sensitivity function after 12week treatment. Boxes indicate the quarter values (from one-fourth to three-fourths); whiskers represent the maximum and minimum value; the hyphen in the box indicates the median value; the cross in the box indicates the mean value. ${ }^{*} p<0.05$ compared with the improvement in contrast sensitivity function in the patching group.

\section{Stereoacuity}

After treatment, the mean Titmus stereoacuity significantly improved to $210.00 \pm 185.19(95 \% \mathrm{CI}=123.33-$ 296.67) arc seconds and $105.00 \pm 75.57$ (95\% CI $=69.63-$



Fig. 4. Stereoacuity at baseline and each follow-up visit. Boxes indicate the mean value; whiskers represent the $95 \%$ confidence interval; ${ }^{*} p<0.05$ compared with the baseline stereoacuity in the AFG group.

140.37) arc seconds at the visits of 3 and 12 weeks in the AFG group when compared with the baseline (609.00 \pm $860.54[95 \% \mathrm{CI}=206.26-1,011.74]$ arc seconds, $p<0.05$, respectively; shown in Fig. 4). In the patching group, the 
stereoacuity was $576.00 \pm 637.15(95 \% \mathrm{CI}=277.80$ 874.20 ) arc seconds at the baseline. At the follow-up visits of 3 and 12 weeks, the stereoacuity had improved to $407.00 \pm 262.24(95 \% \mathrm{CI}=284.27-529.73)$ arc seconds $(p=0.15$, shown in Fig. 4$)$ and $312.50 \pm 276.88$ (95\% CI $=182.92-442.08)$ arc seconds $(p=0.08$, shown in Fig. 4$)$. None of the subjects stated significant uncomfortable symptoms including headache, eyestrain, or dizzy and nausea after wearing the AFG or the patch in this study.

\section{Discussion}

The initial result of the AFG therapy was very encouraging with the significant improvement in both monocular and binocular visual function. Additionally, the CSF of high spatial frequency had got an improvement after AFG treatment. This study suggested that $1 \mathrm{~h}$ of $7-\mathrm{Hz}$ alternative occlusion is effective in treating anisometropic amblyopia, which was not inferior to $2 \mathrm{~h}$ of occlusion.

In our study, we found improvement in BCVA after AFG treatment with $7 \mathrm{~Hz}$. At the alternative flicker rate of $7 \mathrm{~Hz}$, subjects presented with fusion or superimposition of the images because the time course of interocular rivalry suppression in amblyopic subjects was longer than $150 \mathrm{~ms}$ [14]. Therefore, the $7-\mathrm{Hz}(143 \mathrm{~ms})$ alternation frequency of AFG fulfilled the requirement for an antisuppression therapy with bilateral sight [15]. Eric [16] once used a similar electronic rapid alternate occlusion with liquid crystal lenses flicking at $5 \mathrm{~Hz}$ to treat intermittent central suppression, which was effective in reducing suppression within the cortex. In this study, the improvement in BCVA in the AFG group was not inferior to the patching group although the occlusion time is much less. This proved that binocular treatment could also have an effect on monocular visual acuity. Additionally, the subjects were mainly older children, who were beyond the critical period of visual development in this study. This may suggest that the visual acuity of amblyopia in older children still had the potential to recover [17]. Previous studies had also applied liquid crystal glass to the treatment of amblyopia and found improvement in visual acuity [18-20]. But, it was different from this study. In most studies, liquid crystal glass has been applied monocularly with the same aim as patching therapy, to improve visual acuity of the amblyopic eye. Wang and his colleagues [18] used flicker glasses, set at 30-s opaque/transparent intervals for $4 \mathrm{~h}$ a day, to treat amblyopia. Spierer et al. [19] demonstrated that liquid crystal glass was effective in the treatment of amblyopia by using the procedure of $40-\mathrm{s}$

A New Anti-Suppression Approach to the

Treatment of Amblyopia occlusion and 20-s open of each minute with $5 \mathrm{~h}$ of occlusion in a day. Therefore, it may not have an effect on the binocular fusion. This study could give insight into the efficacy of 7-Hz AFG in improving amblyopic eye visual acuity in older children.

We found that AFG therapy could improve the CSF of the amblyopic eye, including both the low and high spatial frequencies, which was different from the occlusion treatment. Before the treatment, the deficit of the CSF in anisometropic amblyopia crossed all ranges of spatial frequencies, which was consistent with previous studies [21, 22]. According to Nicholas et al. [23], the CSF is a basic characteristic of the visual system and the most important recording in spatial vision. A previous study demonstrated that the monocular contrast sensitivity might be associated with the binocularity of neurons in the early visual pathway [24]. Treatment for binocular functions may be necessary for the recovery of monocular spatial vision. It was reported that the CSF of the amblyopic eye improved after the perceptual learning or vernier task, which could diminish the cortical suppression [25-27]. At the third week, there was a significant improvement in the CSF of 3,6 , and 12 cycles per degree spatial frequencies in the AFG group, while only in the 3 cycles per degree spatial frequency CSF in the patching group. The improvement of 18 cycles per degree spatial frequency was insignificant in both groups. It is known that higher spatial frequencies of the CSF develop very quickly in infancy and mature by 3-6 years of age, while lower spatial frequencies mature around the ages of 9-12 years $[28,29]$. Therefore, the recovery of high spatial frequency may require longer observation and more treatment. With the extension of the observation time, the CSF of 18 cycles per degree spatial frequency also presented a significant improvement in the AFG group and still insignificant improvement in the patching group. This indicated that binocular treatment may have advantage in recovering high spatial frequency contrast sensitivity. However, the high spatial frequency of the CSF in the amblyopic eyes still had some deficits compared with the fellow eyes after 12-week treatment. The signal of high spatial frequency was thought to be input from the parvocellular pathway, while the low spatial frequency was from the magnocellular pathway [21]. The residual deficits in the high spatial frequency may be associated with the mis-wiring of the cortical neurons in the visual processing or downweighting of the high spatial frequency channels of the amblyopic eyes.

The stereoacuity significantly improved after AFG treatment in this study. It agreed with previous report that binocular treatment could induce stereoacuity re- 
covery $[30,31]$. Additionally, an interesting finding in our study was that the improvement in the stereoacuity was not parallel to the improvement in the visual acuity in the AFG group. One subject did not have improvement in the visual acuity, although the stereoacuity reached 140 arc seconds. Conversely, we found that there is no significant improvement in stereoacuity, although the visual acuity significantly improved in the patching group. This indicated that AFG treatment led to recovery of the binocular function first, which was different from the occlusion treatment [32]. Taking together, it suggested that binocular fusion training, placing the images on corresponding areas in the 2 eyes, may provide a useful scaffold for integrating signals from the 2 eyes and therefore present a more efficient way in recovery of stereovision in amblyopic patients [33]. It would be interesting to combine the different monocular and binocular therapy and verify if recovery of stereoacuity and visual acuity is truly independent in further study.

There were several limitations in this study. The visual function of the majority of subjects still had deficits at the last follow-up visit. We are not clear whether there is regression in visual acuity when the treatment is stopped. In this pilot study, the sample sizes were roughly small. Large-scale, randomized clinical trials are needed in the future. Additionally, this study did not involve all types of amblyopia, such as strabismus amblyopia, which is thought to have a greater degree of suppression than anisometropic amblyopia. Further study will focus on the efficacy of the AFG in both anisometropic and strabismus amblyopia.

\section{Conclusion}

AFG was a promising tool for treating anisometropic amblyopia, which could improve not only the monocular visual function but also the binocular visual function. Our results provided some insightful information for improving our understanding of the underlying suppression mechanism of anisometropic amblyopia and adding a new approach to the treatment strategy.

\section{Acknowledgment}

The authors thank Min Li for her valuable support during data collection.

\section{Statement of Ethics}

The study protocol complied with the requirements of the Institutional Review Board of Shanghai General Hospital, Shanghai Jiao Tong University School of Medicine (IRB No. 2016KY229) and adhered to the tenets of the Declaration of Helsinki. Informed written consent was obtained from the subjects and at least one of the subject's legal guardians before participation. The trial registration number is NCT02970708, Registered November 17, 2016.

\section{Conflict of Interest Statement}

The authors have no conflicts of interest to declare.

\section{Funding Sources}

This work was supported by Grant 2020YFC2003904 from the National Key Research \& Development Program, Grant 16CR3032A from the Shanghai Shenkang Development Center Project, Grant 81770953 from the National Natural Science Foundation, Grant 2018ZHYL0222 from the intelligent medical project of Shanghai, and Grant 17411950204 from the Science and Technology Commission of Shanghai Municipality. Grant CTCCR2018B01 from Clinical Research Innovation Plan of Shanghai General Hospital, Grant 82070992 from National Natural Science Foundation, Grant 81900900 from National Natural Science Foundation, Grant ZH2018QNA18 from Translational Medicine Crossover Research Fund of Shanghai Jiao Tong University.

\section{Author Contributions}

B.K. and Y.Y. were involved in study design; Y.Y., C.Z., P.W., X.H., W.Y., X.H., and B.K. were involved in collection, management, and interpretation of data; Y.Y. and B.K. were involved in data analysis and writing of the article; Y.Y. and B.K. were involved in preparation, review, and approval of the manuscript; Y.Y. contributed to the manuscript as the first author; B.K. contributed to the manuscript as the corresponding author. All authors read and approved the final manuscript.

\section{Data Availability Statement}

The data that support the findings of this study are not publicly available due to their containing information that could compromise the privacy of research participants. However, they are available from the corresponding author upon reasonable request. 


\section{References}

1 Repka MX, Kraker RT, Holmes JM, Summers AI, Glaser SR, Barnhardt CN, et al. Atropine vs patching for treatment of moderate amblyopia: follow-up at 15 years of age of a randomized clinical trial. JAMA Ophthalmol. 2014 Jul;132(7):799-805.

2 Huang C, Tao L, Zhou Y, Lu ZL. Treated amblyopes remain deficient in spatial vision: a contrast sensitivity and external noise study. Vision Res. 2007 Jan;47(1):22-34.

3 Wallace DK, Lazar EL, Melia M, Birch EE, Holmes JM, Hopkins KB, et al. Stereoacuity in children with anisometropic amblyopia. J AAPOS. 2011 Oct;15(5):455-61.

4 Bhola R, Keech RV, Kutschke P, Pfeifer W, Scott WE. Recurrence of amblyopia after occlusion therapy. Ophthalmology. 2006 Nov; 113(11):2097-100.

5 Birch EE. Amblyopia and binocular vision. Prog Retin Eye Res. 2013 Mar;33:67-84.

6 Searle A, Norman P, Harrad R, Vedhara K. Psychosocial and clinical determinants of compliance with occlusion therapy for amblyopic children. Eye. 2002 Mar;16(2):150-5.

7 Li J, Thompson B, Lam CS, Deng D, Chan LY, Maehara G, et al. The role of suppression in amblyopia. Invest Ophthalmol Vis Sci. 2011 Jun 13;52(7):4169-76.

8 Hess RF, Mansouri B, Thompson B. A new binocular approach to the treatment of amblyopia in adults well beyond the critical period of visual development. Restor Neurol Neurosci. 2010;28(6):793-802.

9 Hess RF, Mansouri B, Thompson B. A binocular approach to treating amblyopia: antisuppression therapy. Optom Vis Sci. 2010 Sep;87(9):697-704.

10 Gambacorta C, Nahum M, Vedamurthy I, Bayliss J, Jordan J, Bavelier D, et al. An action video game for the treatment of amblyopia in children: a feasibility study. Vision Res. 2018 Jul;148:1-14

11 Chen Z, Li J, Liu J, Cai X, Yuan J, Deng D, et al. Monocular perceptual learning of contrast detection facilitates binocular combination in adults with anisometropic amblyopia. Sci Rep. 2016 Feb;6:20187.
12 Mower GD, Christen WG. Evidence for an enhanced role of GABA inhibition in visual cortical ocular dominance of cats reared with abnormal monocular experience. Brain Res Dev Brain Res. 1989 Feb 1;45(2):211-8.

13 Holopigian K, Blake R, Greenwald MJ. Clinical suppression and amblyopia. Invest Ophthalmol Vis Sci. 1988 Mar;29(3):444-51.

14 Belsunce S, Sireteanu R. The time course of interocular suppression in normal and amblyopic subjects. Invest Ophthalmol Vis Sci. 1991 Aug;32(9):2645-52.

15 Hussey ES. The on-switch for seeing. J Optom Vis Devel. 2003;34:75-82.

16 Hussey ES. Remote treatment of intermittent central suppression improves quality-of-life measures. Optometry. 2012 Jan;83(1):19-26.

17 Astle AT, Webb BS, McGraw PV. Can perceptual learning be used to treat amblyopia beyond the critical period of visual development? Ophthalmic Physiol Opt. 2011 Nov; 31(6):564-73

18 Wang J, Neely DE, Galli J, Schliesser J, Graves A, Damarjian TG, et al. A pilot randomized clinical trial of intermittent occlusion therapy liquid crystal glasses versus traditional patching for treatment of moderate unilateral amblyopia. J AAPOS. 2016 Aug; 20(4):326-31.

19 Spierer A, Raz J, Benezra O, Herzog R, Cohen E, Karshai I, et al. Treating amblyopia with liquid crystal glasses: a pilot study. Invest Ophthalmol Vis Sci. 2010 Jul;51(7):3395-8.

20 Erbağc1 I, Okumuş S, Öner V, Coşkun E, Çelik $\mathrm{O}$, Ören B. Using liquid crystal glasses to treat ambyopia in children. J AAPOS. 2015 Jun; 19(3):257-9.

21 Zele AJ, Pokorny J, Lee DY, Ireland D. Anisometropic amblyopia: spatial contrast sensitivity deficits in inferred magnocellular and parvocellular vision. Invest Ophthalmol Vis Sci. 2007 Aug;48(8):3622-31.
22 Harwerth RS, Smith EL, Boltz RL, Crawford ML, von Noorden GK. Behavioral studies on the effect of abnormal early visual experience in monkeys: spatial modulation sensitivity. Vision Res. 1983;23(12):1501-10.

23 Nicholas JJ, Heywood CA, Cowey A. Contrast sensitivity in one-eyed subjects. Vision Res. 1996 Jan;36(1):175-80.

24 McKee SP, Levi DM, Movshon JA. The pattern of visual deficits in amblyopia. J Vis. 2003;3(5):380-405.

25 Zhou Y, Huang C, Xu P, Tao L, Qiu Z, Li X, et al. Perceptual learning improves contrast sensitivity and visual acuity in adults with anisometropic amblyopia. Vision Res. 2006 Mar; 46(5):739-50.

26 Levi DM, Polat U, Hu YS. Improvement in vernier acuity in adults with amblyopia. Practice makes better. Invest Ophthalmol Vis Sci. 1997 Jul;38(8):1493-510.

27 Li J, Spiegel DP, Hess RF, Chen Z, Chan LY, Deng $D$, et al. Dichoptic training improves contrast sensitivity in adults with amblyopia. Vision Res. 2015 Sep;114:161-72.

28 Adams RJ, Courage ML. Using a single test to measure human contrast sensitivity from early childhood to maturity. Vision Res. 2002 Apr;42(9):1205-10.

29 Xu P, Lu ZL, Qiu Z, Zhou Y. Identify mechanisms of amblyopia in Gabor orientation identification with external noise. Vision Res. 2006 Oct;46(21):3748-60.

30 Hess RF, Thompson B, Black JM, Machara G, Zhang P, Bobier WR, et al. An iPod treatment of amblyopia: an updated binocular approach. Optometry. 2012 Feb 15;83(2):87-94.

31 Ding J, Levi DM. Recovery of stereopsis through perceptual learning in human adults with abnormal binocular vision. Proc Natl Acad Sci U S A. 2011 Sep 13;108(37):E733-41.

32 Stewart CE, Wallace MP, Stephens DA, Fielder AR, Moseley MJ; MOTAS Cooperative. The effect of amblyopia treatment on stereoacuity. J AAPOS. 2013 Apr;17(2):166-73.

33 Levi DM, Knill DC, Bavelier D. Stereopsis and amblyopia: a mini-review. Vision Res. 2015 Sep;114:17-30. 